\title{
DETERMINATION OF MATERIAL PARAMETERS FOR MICROBUCKLING ANALYSIS OF FIBER REINFORCED POLYMER MATRIX COMPOSITES
}

\author{
M. ROMANOWICZ \\ Department of Mechanical Engineering \\ Bialystok University of Technology \\ Wiejska 45C, 15-351 Bialystok, POLAND \\ E-mail:m.romanowicz@pb.edu.pl
}

\begin{abstract}
This research focuses on studying the effect of the constitutive law adopted for a matrix material on the compressive response of a unidirectional fiber reinforced polymer matrix composite. To investigate this effect, a periodic unit cell model of a unidirectional composite with an initial fiber waviness and inelastic behavior of the matrix was used. The sensitivity of the compressive strength to the hydrostatic pressure, the flow rule and the fiber misalignment angle were presented. The model was verified against an analytical solution and experimental data. Results of this study indicate that a micromechanical model with correctly identified material parameters provides a useful alternative to theoretical models and experimentation.
\end{abstract}

Key words: polymer-matrix composites, buckling, micro-mechanics, finite element analysis.

\section{Introduction}

Computational micromechanics is used as a tool to predict the macroscopic properties of composite materials based on the known properties of their constituents. To avoid considering all the fibers included in a composite, micromechanics uses the concept of a unit cell. A unit cell is the smallest portion of the heterogenous material that contains all geometrical features of the microstructure. Micromechanics is applicable only if microscopic boundary conditions imposed on the unit cell are controlled by the macroscopic quantities, such as macroscopic strain. In the present paper, this condition is fulfilled by applying periodic boundary conditions. The effective properties of the heterogeneous material are calculated by the direct average method. This method is based on volume average of the fields quantities like strain and stress on the microscopic level.

One of the design limiting features of a unidirectional composite lamina at the macroscopic level is the compressive failure in the fiber direction. The compressive strength of modern composites ranges between $50-60 \%$ of their tensile strength. Experimental results indicate that it is triggered by microbuckling of fibers inside the inelastic matrix. Thus, the key parameters of compressive models are the matrix ductility and the initial waviness of fibers. Following the usual assumption that the induced shear strain $\gamma$ and the fiber misalignment angle $\Theta$ are small, simple equilibrium considerations (Budiansky and Fleck, 1993) relate the applied compressive stress $\sigma$ to the shear response of the composite $\tau(\gamma)$ by

$$
\sigma=\tau(\gamma) /(\gamma+\Theta)
$$

This relationship demonstrates that the shear mode failure will always occur during the buckling process. 
Application of a representative unit cell approach in modeling the microbuckling has been reported by Guynn et al. (1992), Morais (1996), Hsu et al. (1999), Pansart et al. (2009) and Gutkin et al. (2010). In all of these papers, the nonlinear constitutive matrix behavior was described by using the von Mises yield criterion. Unfortunately, such an approach does not take into account the pressure sensitivity plastic response of polymer matrices.

The present work attempts to meet this demand. A micromechanical finite element model that incorporates the pressure sensitivity yielding is used to determine the compressive strength of a fiber reinforced polymer composite and to investigate the effect of the above mentioned parameters.

\section{Periodic unit cell model}

\subsection{Initial fiber waviness}

An initial fiber imperfection is uniformly distributed across the width of the model (Fig.1a). For the presentation of the problem, the material coordinate system is introduced so that the $x_{l}$ axis is aligned with the fiber direction and the $x_{2}$ axis is perpendicular to the fiber direction. In this paper, the imperfection is expressed as a sinusoidal waviness of fibers along the $x_{l}$ direction with amplitude $a$ and wavelength $2 \lambda$

$$
v_{0}=a \cos \left(\pi x_{1} / \lambda\right)
$$

If the sinusoidal imperfection has long wavelength and relatively small amplitude, the fiber misalignment angle can be approximated by

$$
\Theta=\pi a / \lambda .
$$

In the literature (see for example Yurgatis, 1987; Fleck, 1997), the values of the initial fiber misalignment $\Theta$ are usually $2^{\circ}-3^{\circ}$. Unit cell models with the uniformly distributed imperfection characterized by $\Theta=1.71^{\circ}, 2.40^{\circ}$ and $3.42^{\circ}$ were analyzed.

a)

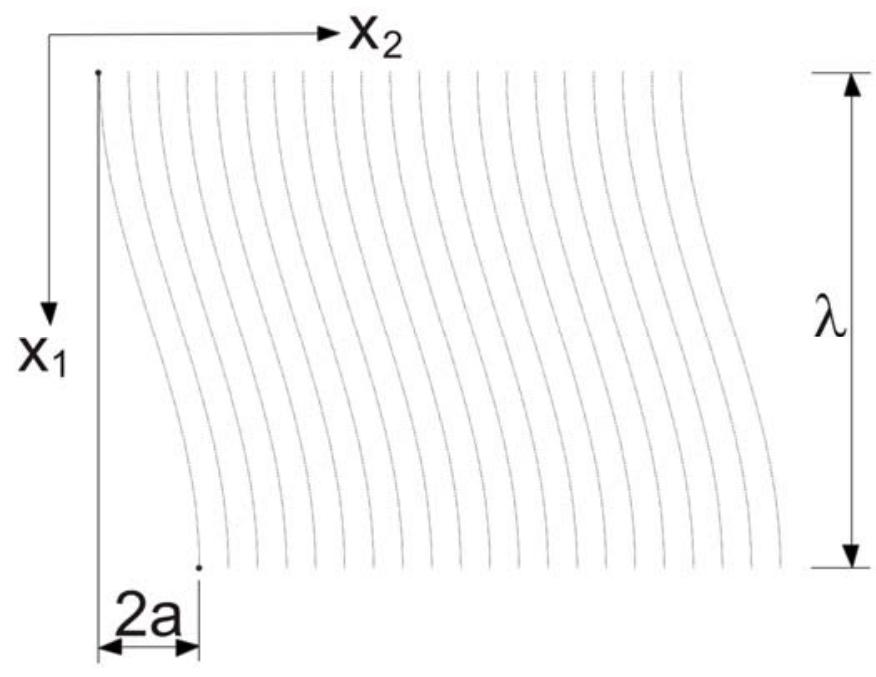


b)

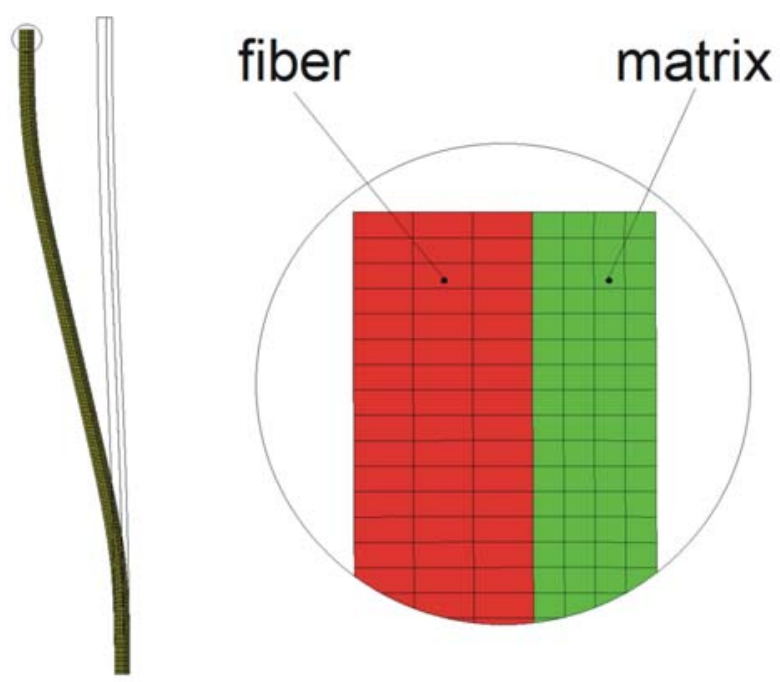

Fig.1. Periodic unit cell model. (a) geometry; (b) finite element mesh.

\subsection{Finite element formulation}

Finite element models are constructed by using ANSYS finite element code. Two-dimensional finite element meshes made of plane strain, biquadratic elements with eight nodes (Plane183) are generated for the appropriate geometries of micromechanical models (Fig.1b). The fiber and matrix layers each have 1575 and 2100 elements, respectively. The width of the matrix region was chosen so as to accommodate the fiber diameter $d_{\mathrm{f}}=7 \mu \mathrm{m}$, and respect the fiber volume content $V_{f}=60 \%$. The length of models corresponds to the half wavelength, $\lambda=75 d_{f}$.

The longitudinal compression is imposed by means of a vertical pressure prescribed at the top side of the unit cell in such a way that the top side remains plane. The bottom side of the unit cell is fixed in the vertical direction. One node was also fixed in the direction perpendicular to the compressive load to prevent rigid body motion.

The periodicity conditions on the right and left sides of the unit cell models are defined to represent a periodic microstructure consisting of an infinitely large array of repeated unit cells. The displacements of nodes on the right side of the unit cell are related to the displacements of their counterparts on the left side as follows (Pansart et al., 2009)

$$
u_{i}^{n 2}-u_{i}^{r e f 2}=u_{i}^{n 1}-u_{i}^{r e f 1} \quad \text { for } \quad i=1,2
$$

where $u_{i}$ is the displacement in the $i$-axis direction, $n_{1}$ and $n_{2}$ is a pair of nodes facing each other on edge 1 and edge 2 , respectively, $\operatorname{ref}_{l}$ and $\operatorname{ref}_{2}$ is a pair of reference nodes on edge 1 and edge 2 .

In order to handle the potential snap-through associated with the post-buckling response effectively, the arc-length method (Riks, 1979) is adopted. A minimum arc-length radius was defined by using the following parameters. The minimum multiplier of reference arc-length radius was $r_{\min }=10^{-8}$. The number of substeps was $n=2500$. A residual forces tolerance of $t o l_{\mathrm{F}}=0.001$ was selected to control the convergence of the iterative procedure. 


\subsection{Constitutive models of fibers and matrix}

Simulations were carried out for an example case of the AS4/PEEK composite system with the fiber volume content of $60 \%$. The properties of this material and its constituents are listed in Tab.1. For the present problem, the fibers are assumed to be isotropic and linearly elastic solids which are perfectly bonded to the matrix. The matrix is modeled within the framework of the finite deformations as an elasto-plastic solid which hardens isotropically.

Table 1. Mechanical properties of the AS4/PEEK composite and its constituents (Kyriakides et al., 1995).

\begin{tabular}{|c|c|c|c|c|c|c|c|}
\hline \multicolumn{2}{|c|}{ AS4 fiber } & \multicolumn{3}{|c|}{ PEEK matrix } & \multicolumn{3}{|c|}{$\begin{array}{l}\text { AS4/PEEK } \\
\text { composite }\end{array}$} \\
\hline $\begin{array}{c}E_{f} \\
(\mathrm{GPa})\end{array}$ & $v_{f}$ & $\begin{array}{c}E_{\mathrm{m}} \\
(\mathrm{GPa})\end{array}$ & $v_{m}$ & $\begin{array}{c}k \\
(\mathrm{MPa})\end{array}$ & $\begin{array}{c}E_{11} \\
(\mathrm{GPa})\end{array}$ & $\begin{array}{c}\sigma_{c}^{-} \\
(\mathrm{GPa})\end{array}$ & $\begin{array}{c}V_{f} \\
(\%)\end{array}$ \\
\hline 214 & 0.263 & 6.14 & 0.356 & 40 & 128 & 1.21 & 60 \\
\hline
\end{tabular}

The deformation of polymeric materials is highly sensitive to the hydrostatic pressure and plastic flow of these materials can exhibit plastic dilatancy. To address this requirement, the Drucker-Prager plasticity model (Drucker and Prager, 1952), which incorporates the linear dependence on the hydrostatic stress, is used. In terms of the first invariant of stress $I_{l}$ and the second invariant of the deviatoric part of stress $J_{2}$, the yield function is given as

$$
f=\alpha I_{1} / 3+\operatorname{sqrt}\left(J_{2}\right)-k
$$

where $\alpha$ is the pressure sensitivity factor, $k$ is the flow stress of the material under pure shear. The DruckerPrager plasticity model with $\alpha=0,0.1,0.2,0.3$ and $k=40 \mathrm{MPa}$ was used to study the compressive failure of AS4/PEEK composite. Note that if $\alpha=0$, Eq.(2.3) reduces to the von Mises yield function. The plastic strain increment $d \varepsilon^{p}$ is specified through the flow rule

$$
d \varepsilon^{p}=d \lambda(\partial g / \partial \sigma)
$$

where $d \lambda$ is a positive scalar of proportionality which determines the amount of plastic strain and $g$ is a plastic potential which in turn determines the direction of plastic strain. If $g=f$, then the plastic flow is associative and, in turn, the pressure sensitivity and plastic dilatancy are described by the same parameter $\alpha$. If $g \neq f$, then the plastic flow is non-associative and the plastic volume change is controlled by choosing an appropriate parameter $\beta$ in the plastic potential function, i.e., $g=\beta I_{1} / 3+\operatorname{sqrt}\left(J_{2}\right)$.

\subsection{Calibration of the Drucker-Prager plasticity model}

In this paper, the in-situ axial stress-strain curve of the matrix is back-calculated from the shear stress-strain curve of the AS4/PEEK composite by using numerical homogenization. To simulate in-plane shear loading, the same two-dimensional unit cell model as the one described in section 2.2 was used. Comparisons of the in-situ axial response of the matrix and the predicted shear response of the composite with corresponding test data (Kyriakides et al., 1995) are shown in Figs 2a and 2b. It can be seen from Fig. $2 \mathrm{~b}$ that the axial stress-strain curves of the matrix shown in Fig.2a produce an accurate solution for the AS4/PEEK composite under in-plane shear loading. 
a)

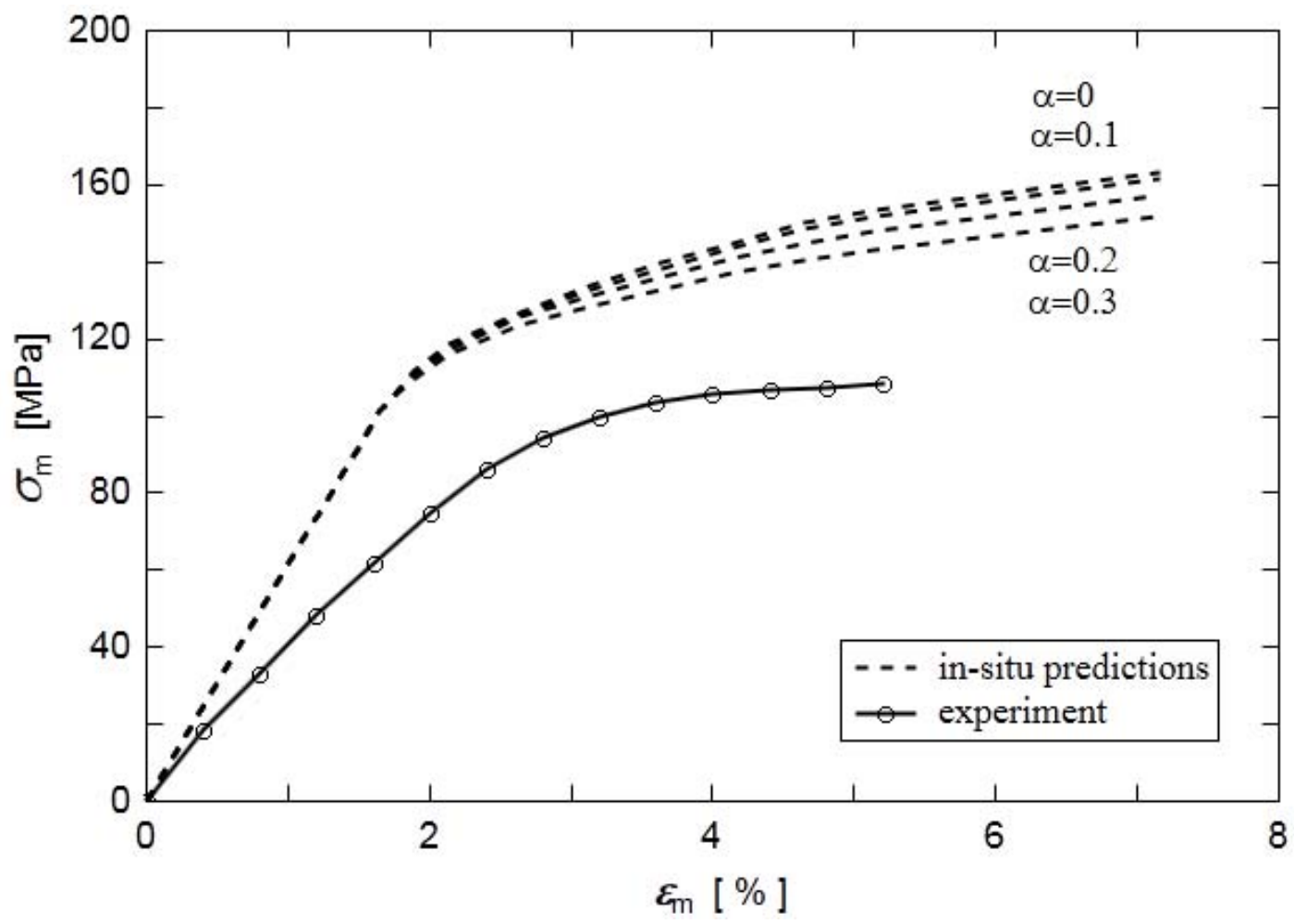

b)

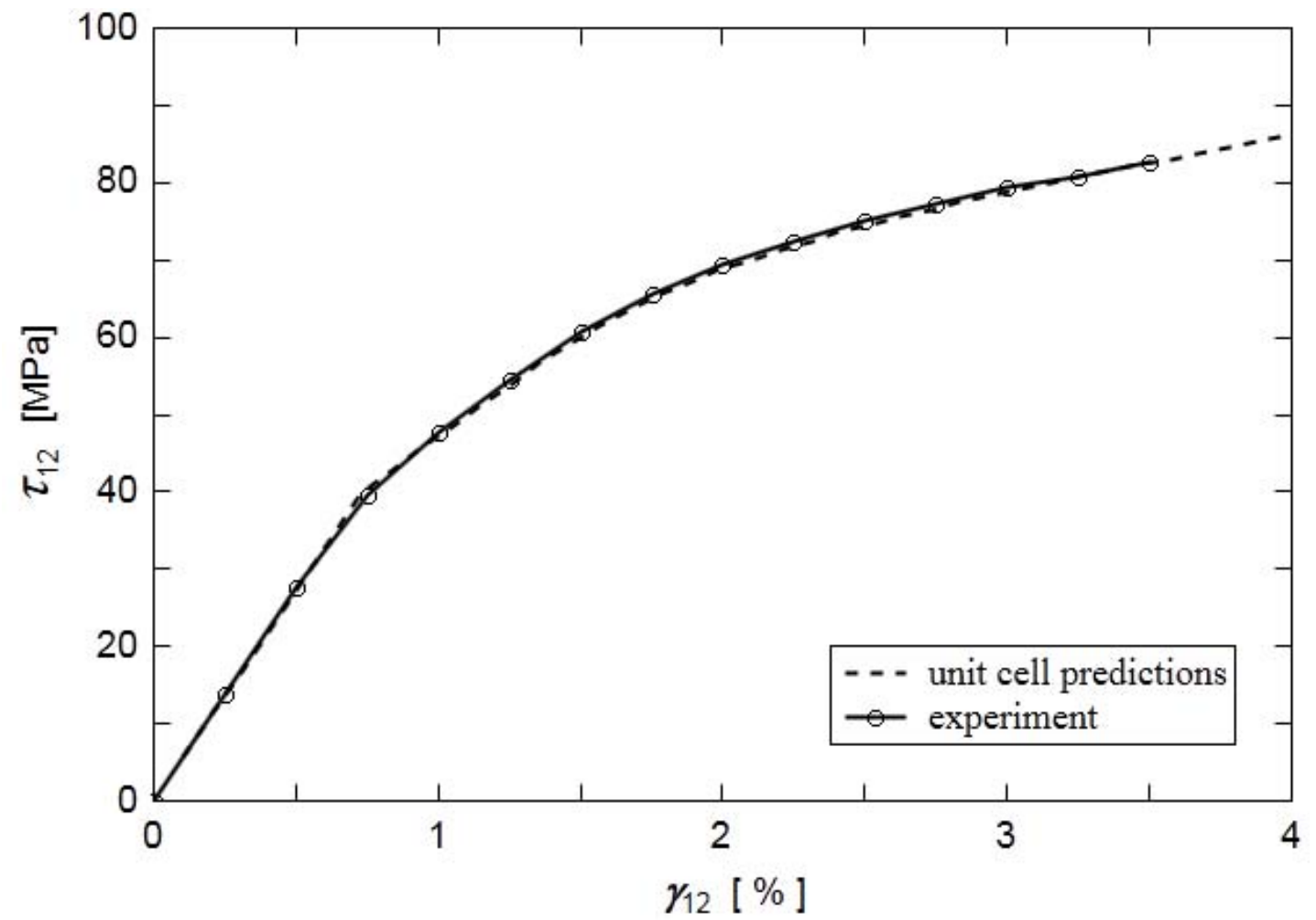

Fig.2. Identification of the plasticity model. (a) comparison of the in-situ axial responses of PEEK matrix with test data (Kyriakides et al., 1995); (b) adjusting experimental shear response of the AS4/PEEK composite (Kyriakides et al., 1995) with model responses. 


\subsection{Numerical homogenization}

The compressive responses of the periodic unit cell models are discussed in terms of macroscopic stress and strain. According to the homogenization theory, the macroscopic compressive stress $\sigma_{11}$ is equal to the average normal traction at the bottom edge of the model (obtained as the sum of reactions divided by the actual cross-section). The corresponding average strain $\varepsilon_{11}$ was computed from a logarithmic formula, namely $\varepsilon=\ln (1+\delta / \lambda)$, where $\delta$ is the displacement of nodes at the top edge of the model.

\section{Results and discussion}

The combination of nonlinear matrix behavior and initial fiber waviness into one micromechanical model enables the determination of the overall response of composites subjected to compressive loading. The macroscopic stress-strain curves calculated for three values of the initial fiber misalignment $\Theta=1.71^{\circ}, 2.40^{\circ}$ and $3.42^{\circ}$ are shown in Figs 3a-3c. The nonlinear responses of the AS4/PEEK composite under longitudinal compression for each angle $\Theta$ are predicted for four values of the pressure sensitivity factor $\alpha=0,0.1,0.2$ and 0.3 . Generally, the main features of the predicted response are similar to those seen experimentally (Kyriakides et al., 1995; Moran et al., 1995). It can be seen that the initial deformation of the composite is linear elastic. The limit load is reached shortly after non-linearity is first noted. After the maximum of the compressive stress has been attained, geometric softening associated with the fiber bending outweighs the strain hardening of the matrix, and the load begins to drop. As expected, the critical stress predicted by the periodic unit cell model is sensitive to the fiber misalignment angle $\Theta$. An increase of the value of $\Theta$ reduces the compressive strength of the composite as well as the snap-through instability beyond the limit load. On the other hand, an increase of the value of $\alpha$ improves the resistance of the composite to compressive failure. This finding clearly indicates that the application of a matrix with high internal friction during composite processing should provide better failure characteristics. It is interesting to observe that the use of the von Mises plasticity model $(\alpha=0)$ leads to lower compressive strengths compared to the Drucker-Prager plasticity model $(\alpha>0)$.

a)

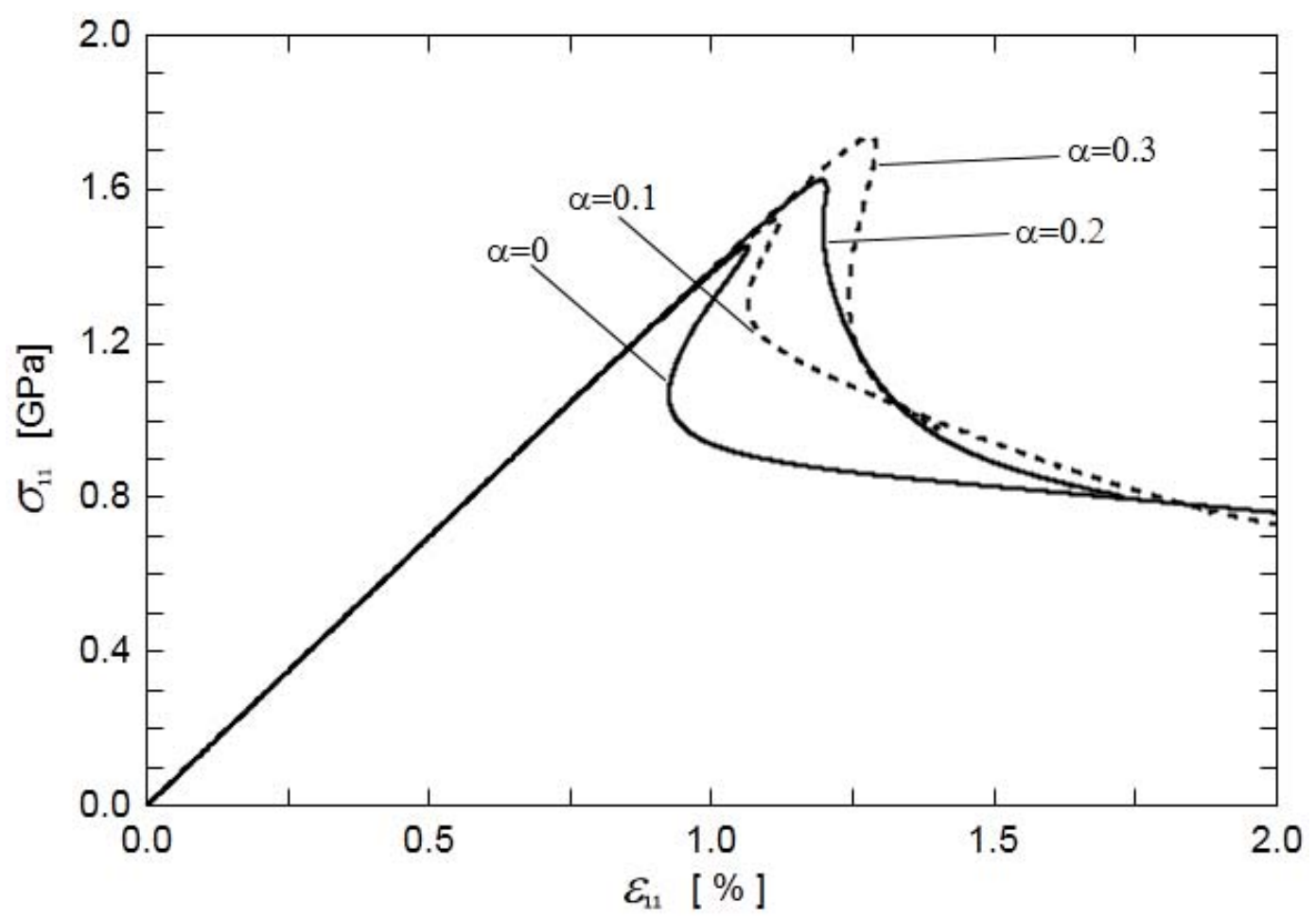


b)

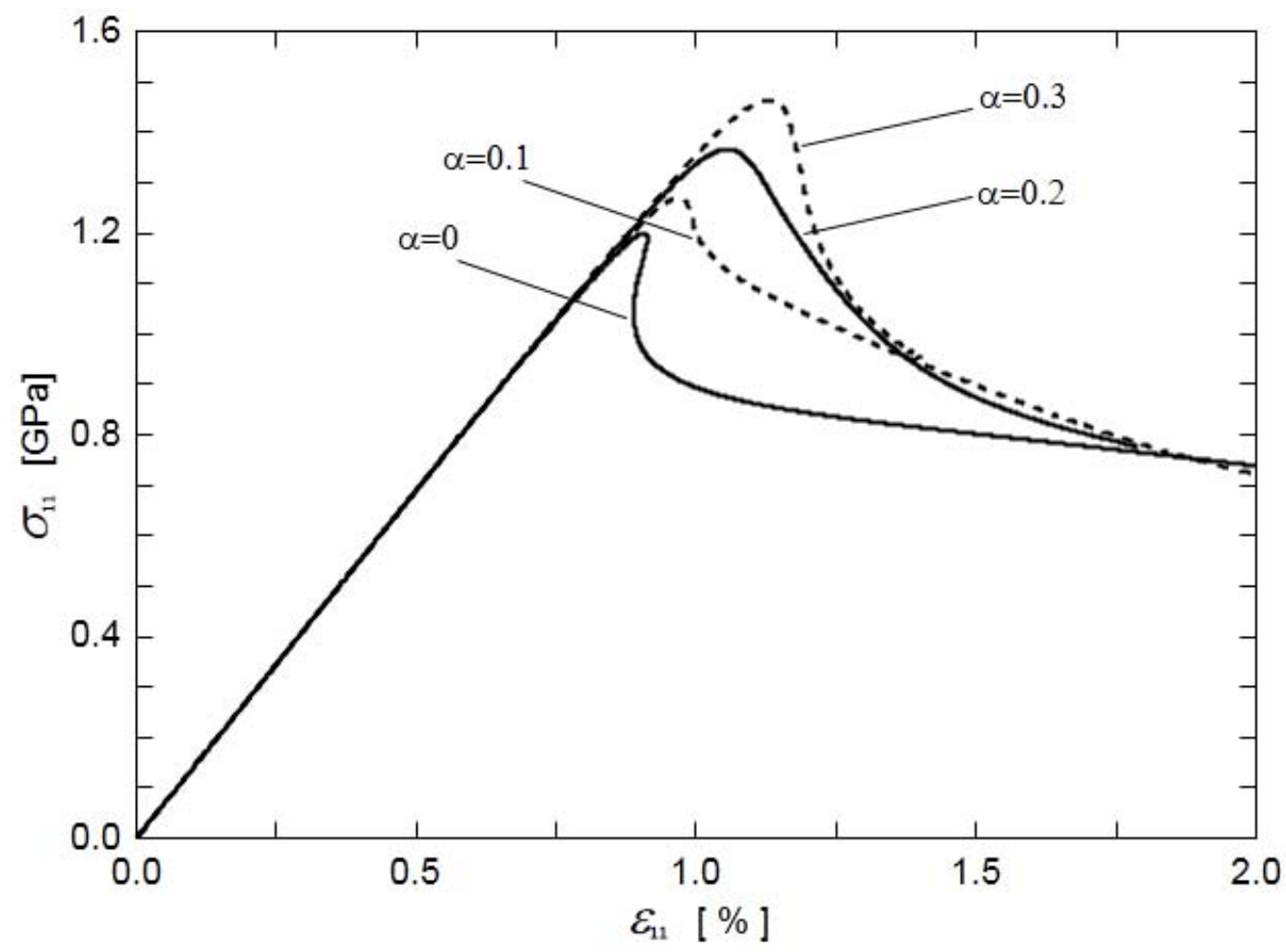

c)

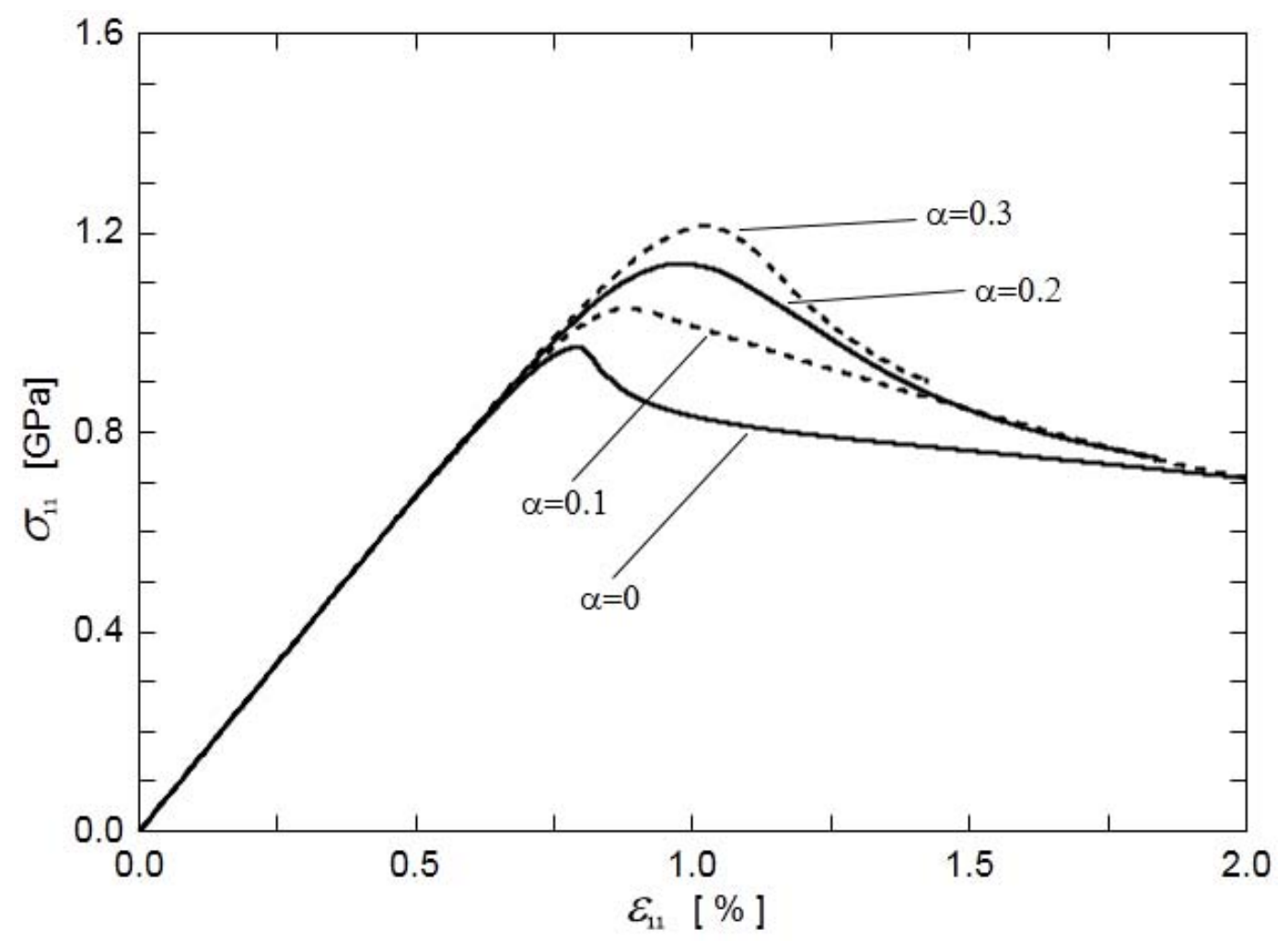

Fig.3. Prediction of the compressive stress-strain curves for various values of the pressure sensitivity factor $\alpha$. Responses for (a) $\Theta=1.71^{\circ}$; (b) $\Theta=2.40^{\circ}$; (c) $\Theta=3.42^{\circ}$. 
In order to assess the effect of the flow rule on the compressive response of the AS4/PEEK composite, the macroscopic stress-strain curves calculated for one value of the pressure sensitivity factor $\alpha=0.1$ through the model in which the matrix followed the associative flow rule were compared to the corresponding curves obtained from the non-associative flow rule. The results of this comparison are summarized in Fig.4 for three values of the initial fiber misalignment $\Theta$. The non-associative curves relate to the limiting case, $\beta=0$ in which no volume change during plastic deformation takes place. It can be seen that the flow rule has a negligible influence on the onset of buckling. A noticeable difference was only observed beyond the limit load in the post-buckling stage.

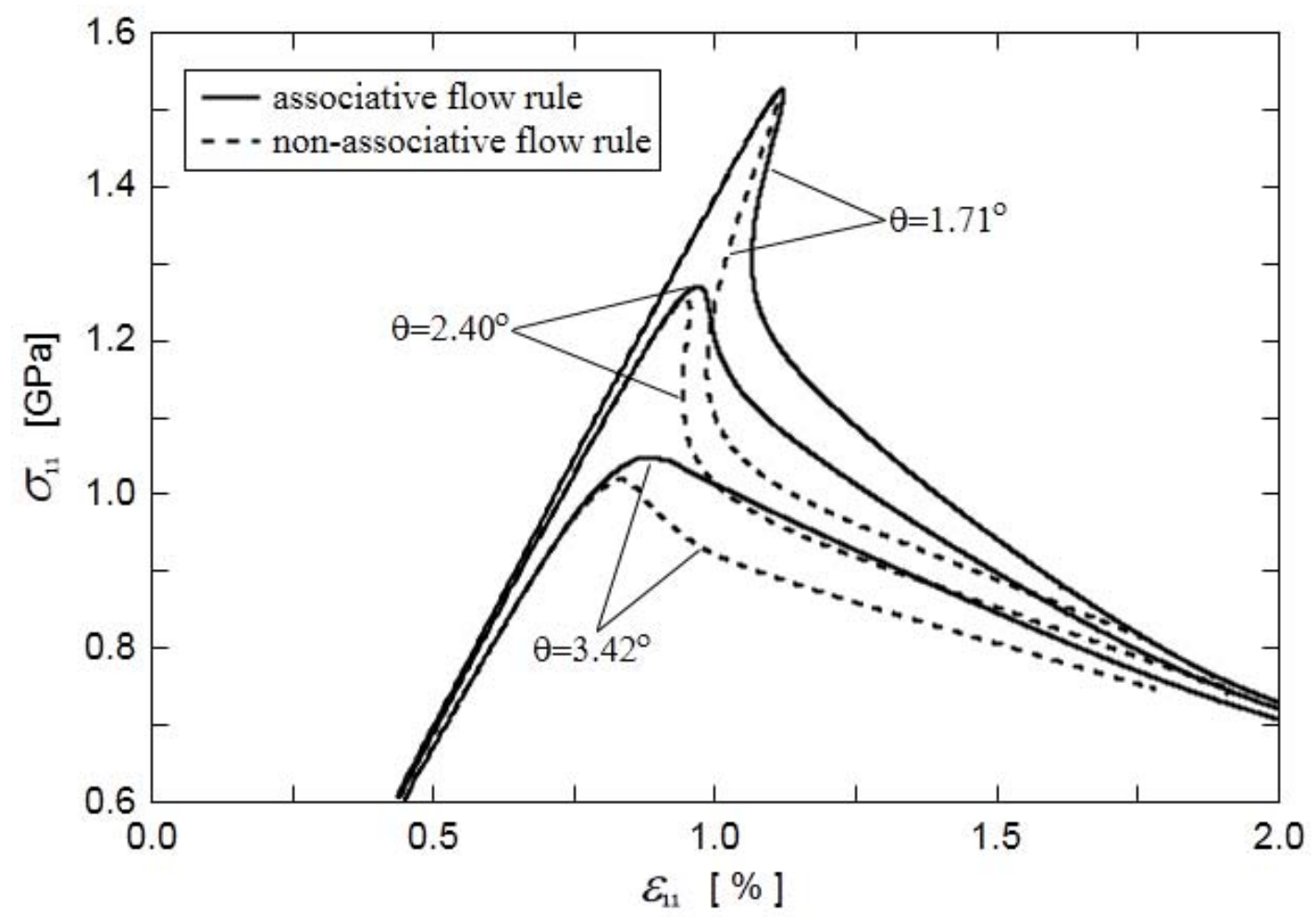

Fig.4. Comparison between the associative $(\alpha=\beta=0.1)$ and non-associative $(\alpha=0.1, \beta=0)$ flow rule for various values of the fiber misalignment angle $\Theta$.

In order to determine material parameters for the microbuckling analysis of the AS4/PEEK composite, the limit stresses obtained through the periodic unit cell models were compared to experimental data reported by Kyriakides et al. (1995) as well as to predictions made with theories proposed by Budiansky and Fleck (1993) and Barbero (1998). The first analytical formulation is based on the physics of kink-band formation and the other on the stability analysis. Both theories take into account the matrix physical nonlinearity and the presence of initial fiber misalignment. The Budiansky and Fleck theory suggests that the longitudinal compressive strength $\sigma_{c}$ can be derived as

$$
\sigma_{c}=G\left[1+\left(\sigma_{t y} / \tau_{y}\right)^{2} \tan ^{2} \beta\right] /\left[1+n(3 / 7)^{1 / n}\left[\Theta /(n-1) \gamma^{*}\right]^{n-1 / n}\right]
$$

where $\gamma^{*}=\gamma_{y} /\left[1+\left(\sigma_{t y} / \tau_{y}\right)^{2} \tan ^{2} \beta\right]^{1 / 2}$. In this formula, the ratio of the transverse yield stress to the shear yield stress, $\left(\sigma_{t y} / \tau_{y}\right)=2, \gamma_{y}$ is the shear yield strain, $G$ is the elastic shear modulus of the composite, $n$ 
denotes the strain hardening coefficient, $\Theta$ is the initial misalignment angle and $\beta$ is the kink-band angle. In the Barbero formulation, the longitudinal compressive strength $\sigma_{c}$ is predicted to be

$$
\sigma_{c}=G+\left(16 C_{2} \Theta / 3 \pi\right)-4 \operatorname{sqrt}\left(2 C_{2} \Theta\left(8 C_{2} \Theta+3 \pi G\right)\right) / 3 \pi
$$

where $C_{2}=G^{2} / 4 \tau_{c}$ and $\tau_{c}$ is the in-plane shear strength. The compressive strengths of the AS4/PEEK composite predicted from the analytical formulations were plotted in Fig.5. The predictions are made for the following material constants taken from the shear stress-strain response shown in Fig.2b: $G=5.79 \mathrm{GPa}, \gamma y=1.03 \%, n=4.7, \beta=15^{\circ}, \tau_{c}=80.25 \mathrm{MPa}$.

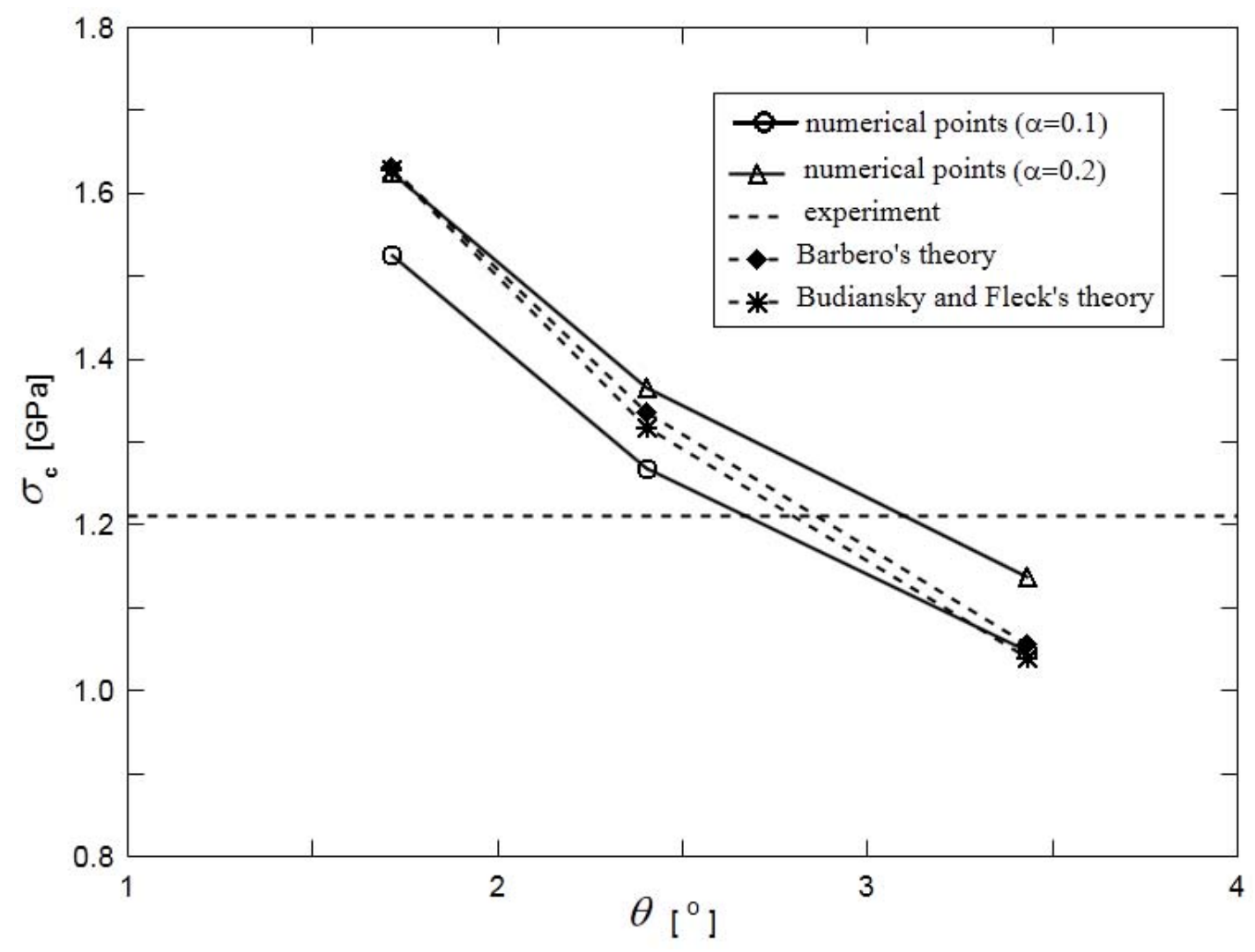

Fig.5. Comparison of the calculated critical stress with experimental data (Kyriakides et al., 1995) and theoretical prediction (Budiansky and Fleck, 1993; Barbero, 1998).

It is interesting to note that the analytical predictions lie between the numerical values of the limit stresses obtained for $\alpha=0.1$ and 0.2. This finding supports the general idea that the von Mises plasticity model $(\alpha=0)$ is unable to appropriately reproduce the deformation of polymeric matrices. Thus, the Drucker-Prager plasticity models with the pressure sensitivity factor $\alpha$ within the range of $0.1-0.2$ are more reliable. By comparing the numerical values of the limit stresses obtained for $\alpha=0.1$ and 0.2 with the mean value of experimental data, it can be concluded that the models with the imperfection parameter $\Theta$ within the range of $2.50^{\circ}-3.25^{\circ}$ guarantee agreement with experiments.

\section{Summary}

A micromechanical finite element analysis has been conducted to investigate the effect of the matrix constitutive behavior and initial fiber waviness on the compressive strength of the unidirectional AS4/PEEK 
composite. It was found that an increase of the value of the misalignment angle reduces the compressive strength of the composite as well as the snap-through instability beyond the limit load. It was also shown that the kind of plasticity model significantly affects the limit load whereas the kind of flow rule is negligible. Furthermore, it was shown that a micromechanical model with correctly identified material parameters provides a useful alternative to theoretical models and experimentation. It was found that micromechanical models with the pressure sensitivity factor $\alpha$ within the range of $0.1-0.2$ and the misalignment angle within the range of $2.50^{\circ}-3.25^{\circ}$ produce the most reliable values of the compressive strength of the unidirectional AS4/PEEK composite.

\section{Acknowledgments}

The financial support of the National Science Centre of Poland under contract DEC2011/03/D/ST8/04817 is acknowledged with gratitude.

\section{Nomenclature}

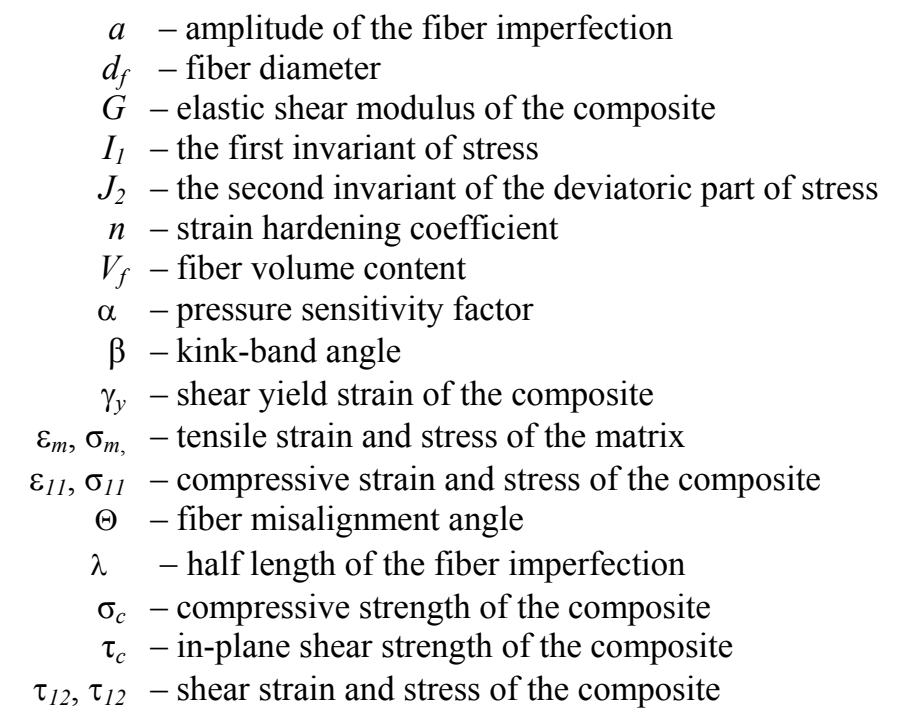

\section{References}

ANSYS users's manual (Version 14). Cannonsburg PA: ANSYS, 2012.

Barbero E.J. (1998): Prediction of compression strength of unidirectional polymer matrix composites. - J. Compos. Mater., vol.32, pp.483-502.

Budiansky B. and Fleck N.A. (1993): Compressive failure of fibre composites. - J. Mech. Phys. Solids, vol.41, pp.183211.

Drucker D.C. and Prager W. (1952): Soil mechanics and plastic analysis for limit design. - Q. App. Math., vol.10, pp.157-165.

Fleck N.A. (1997): Compressive failure of fiber composites. - Adv. Appl. Mech., vol.33, pp.43-117.

Gutkin R., Pinho S.T., Robinson P. and Curtis P.T. (2010): Micro-mechanical modeling of shear-driven fibre compressive failure and of fibre kinking for failure envelope generation in CFRP laminates. - Compos. Sci. Technol., vol.70, No.8, pp.1214-1222.

Guynn E.G., Ochoa O.O. and Bradley W.L. (1992): A parametric study of variables that affect fiber microbuckling initiation in composite laminates: Part I analyses. - J. Comp. Mater., vol.26, No.11, pp.1594-1611. 
Hsu S.Y., Vogler T.J. and Kyriakides S. (1999): On the axial propagation of kink bands in fiber composites: Part II analysis. - Int. J. Solids Structures, vol.36, No.4, pp.575-595.

Jelf P.M. and Fleck N.A. (1994): The failure of composite tubes due to combined compression and torsion. - J. Mater. Sci., vol.29, pp.3080-3084.

Kyriakides S., Arseculeratne R., Perry E.J. and Liechti K.M. (1995): On the compressive failure of fiber reinforced composites. - Int. J. Solids Structures, vol.32, No.6, pp.689-738.

Morais A.B. (1996): Modelling lamina longitudinal compression strength of carbon fibre composite laminates. - J. Compos. Mater., vol.30, No.10, pp.1115-1131.

Moran P.M., Liu X.H. and Shih C.F. (1995): Kink band formation and band broadening in fiber composites under compressive loading. - Acta Metall Mater; vol.43, No.8, pp.2943-2958.

Pansart S., Sinapius M. and Gabbert U. (2009): A comprehensive explanation of compression strength differences between various CFRP materials: Micro-meso model, predictions, parameter studies. - Compos Part A, vol.40, No.4, pp.376-387.

Riks E. (1979): An incremental approach to the solution of snapping and buckling problems. - Int. J. Solids Struct., vol.15, pp.529-551.

Yurgatis S.W. (1987): Measurement of small angle misalignments in continuous fibre composites. - Compos. Sci. Technol., vol.30, pp.279-293.

Received: January 3, 2014

Revised: March 18, 2015 